\title{
Medienproduktion, Medienprodukt und Medienrezeption: Überlegungen zu den medienanalytischen Möglichkeiten der „Rahmentheorie“ und komplementärer Ansätze
}

\author{
Herbert Willems
}

Der Aufsatz bezweckt die Entfaltung eines Apparats komplementärer Konzepte, die es ermöglichen, die Ebenen der Medienproduktion, der Medienprodukte („Medientexte“) und der Medienrezeption als einen Zusammenhang zu betrachten. Auf diese Weise sollen die Nachteile sachlich einseitiger (z. B. nur auf die Seite der Medienrezeption konzentrierter) Medienanalysen vermieden werden. Gegenstand der Untersuchung sind unter anderem die Konzepte Figuration, Feld, Habitus, Semantik und kulturelles Forum. Der Schwerpunkt der Aufmerksamkeit liegt auf der Goffman'schen Rabmentheorie.

Die Überlegungen dieses Aufsatzes ${ }^{1}$ zielen auf Konzepte, die es erlauben, die verschiedenen Ebenen der „Realität der Massenmedien“ (Luhmann) - Medienproduktion, Medienprodukt und Medienrezeption - als einen Zusammenhang zu analysieren. Die damit mögliche Überwindung der bisher vorherrschenden isolierten Betrachtung einzelner Medienebenen - vor allem der „Medientexte“ und neuerdings im Kontext der „Cultural Studies“ (wieder) der Medienrezeption - verspricht nicht nur ein komplexeres, sondern auch ein im Hinblick auf die einzelnen Medienebenen besseres Verständnis der Medienrealität.

Die im Folgenden diskutierten Konzepte und Ansätze sind unterschiedlich abstrakt und ergänzen sich sachlich. Am weitesten reichen die verwandten Konzepte Figuration und Feld. Sie umfassen alle genannten Ebenen und verweisen mit der Einbeziehung der Verhaltensdispositionen der Medienakteure - Medienproduzenten und Medienrezipienten - auf den Habitusbegriff. Die Sinndimension der im Medienkontext relevanten Erlebnisse und Handlungen sowie der Medienkommunikation selbst wird durch die Konzepte Semantik und Rahmen spezifiziert. Insbesondere der Rahmenbegriff, auf dem der Schwerpunkt der Aufmerksamkeit liegt, trägt dazu bei, mediale „Enkodierungen“ und medienrezeptive „Dekodierungen“ zu beschreiben. Die Kulturbezogenheit der Medienkommunikation wird in einem grundlegenden Sinne mit dem Begriff des kulturellen Forums gefasst. Er steht genauer gesagt für das reflexive Verhältnis der Massenmedien zu der kulturellen Umwelt, die sie in ihren Genres verarbeiten und präsentieren. Dieses Verhältnis lässt sich ebenso als Rahmung beschreiben wie das variantenreiche Verhältnis von Medienrezeption und „Medientext“.

Ausgangspunkt und Anstoß der folgenden Untersuchung ist die gegenwärtige Diskussion um den Theatralitätsbegriff, der sich mittlerweile anschickt, zu einem kulturwissenschaftlichen Leitbegriff zu werden. ${ }^{2}$ Unter dem Begriff der Theatralität, der ansatzweise eine komplexe analytische Perspektive in dem obigen Sinne markiert, versteht die Theaterwissenschaftlerin Erika Fischer-Lichte vier Aspekte: „1. den der Performance, die als Vorgang einer Darstellung durch Körper und Stimme vor körperlich an-

1 Für Kritik, Anregung und Hilfestellung danke ich Dr. Ruth Ayaß und York Kautt M. A.

2 Vgl. Fischer-Lichte 1998; Ontrup/ Schicha 1999. 
wesenden Zuschauern gefasst wird [...]; 2. den der Inszenierung, der als spezifischer Modus der Zeichenverwendung in der Produktion zu beschreiben ist; 3. den der Korporalität, der sich aus dem Faktor der Darstellung bzw. des Materials ergibt, und 4. den der Wabrnebmung, der sich auf den Zuschauer, seine Beobachterfunktion und -perspektive bezieht“. ${ }^{3}$ Will man den Theatralitätsbegriff auf die gesellschaftliche Inszenierungspraxis der Medien ausdehnen, dann bedarf es allerdings zweier Modifikationen der von der Theatralität des Theaters ausgehenden Begriffsfassung von Fischer-Lichte. Zum einen ist klar, dass die mediale „performance“ im Gegensatz zu der des Theaters oder des Alltags nicht „als Vorgang einer Darstellung durch Körper und Stimme vor körperlich anwesenden Zuschauern“ zu fassen ist. Die verschiedenen Typen der Medientechnik und die entsprechenden Modi und Stile ihres Gebrauchs stellen vielmehr eigenständige und eigenständig wirklichkeitswirksame Ebenen zwischen „performance“ und Wahrnehmung dar. Zum anderen spielt der Aspekt der Korporalität, als „Leibhaftigkeit“ verstanden, im Rahmen der Massenmedien eine weniger große und andere Rolle als im Theater. Während der Körper und insbesondere die Stimme hier von allergrößter und unverzichtbarer Bedeutung sind (es handelt sich dabei ja um die „Medien“ der Theaterinszenierung) können oder müssen die verschiedenen Massenmedientypen „Leibhaftigkeit" systematisch und spezifisch exklusiv verknappen oder nihilieren. Man denke nur an das Radio, das Korporalität auf die Stimme beschränkt, an die Presse, in der Korporalität potenziell ganz durch die „Materialität“ der Schrift ersetzt wird, oder das Internet, in dessen Kommunikation der Körper gleichfalls gänzlich verschwinden kann. ${ }^{4}$ Der Theatralitätsbegriff muss also entsprechend um- und eingestellt werden. ${ }^{5}$ Darüber hinaus bedarf es einer Einbettung der Theatralitätsperspektive in den im Folgenden entfalteten konzeptuellen Kontext, der seinerseits von den Elementen des Bühnenmodells profitieren kann.

\section{Mediale Praxiskontexte}

Die Analyse massenmedialer Theatralität braucht Konzepte wie das des Dispositivs (Foucault), der Figuration (Elias) oder des Feldes (Bourdieu), weil Medienproduktionen, Medienprodukte und Medienwahrnehmungen (und damit verbundene Verarbeitungen, Verwertungen und Nutzungen) auf Zusammenhänge diverser und mehr oder weniger komplexer sozialer Sinn- und Handlungskontexte verweisen. Unter Dispositiv versteht Schmidt im Medienzusammenhang „eine komplexe Struktur [...], die die Anordnung der Apparate zur Distribution von Medienangeboten, die für die Apparate er-

3 Fischer-Lichte 1998, S. 86.

4 Für das Internet stellt Mike Sandbothe fest, dass dessen „,virtuelle Realität‘ (...) nicht zuletzt deshalb als ,virtuell' bezeichnet wird, weil es sich bei ihr um eine Realitätsform handelt, deren Konstruktion sich in einem künstlichen digitalen Raum vollzieht, in dem die Nutzerinnen und Nutzer losgelöst von den raum-zeitlichen Zwängen ihrer physischen Körperlichkeit virtuelle Körper mit imaginären Eigenzeiten und Eigenräumen erfinden können“ (Sandbothe 1998, S. 587). Die (Internet-)mediale Entbindung von der primären Korporalität des Lebens, die ja Element der obigen Theatralitätsdefinition ist, führt zu einer spezifischen Expansion und Modulation von Theatralität. „Denn im Netz ist infolge der medialen Entkörperlichung seiner Akteure die Kommunikation von den psychosozialen Aspekten einer raum-zeitlich fixierbaren Körperidentität entlastet, die dem theatralen Spiel mit Identitäten im , wirklichen' Leben physische Grenzen setzt" (Sandbothe 1998, S. 588).

5 Vgl. Sandbothe 1998, S. 584ff. 
zeugten Medienangebote sowie die mentale Maschinerie der wahrnehmenden Zuschauer umfaßt ${ }^{*}{ }^{6}$ Der Aspekt der Wahrnehmung ist demnach - als Bedingung der Medienproduktion bzw. der medientheatralen „Textgestaltung“ - in das Konzept des Dispositivs inkludiert. Ausgeschlossen bleibt dagegen die Ebene der Produzenten, deren „mentale Maschinerie“ und praktische Handlungsbedingungen. Die weiter gefassten Begriffe Figuration und Feld schließen diese Aspekte mit ein und sind insofern angemessener und brauchbarer. Unter einem Feld, z. B. dem des Journalismus, versteht Bourdieu Folgendes:

„Ein Feld ist ein strukturierter gesellschaftlicher Raum, ein Kräftefeld - es gibt Herrscher und Beherrschte, es gibt konstante, ständige Ungleichheitsbeziehungen in diesem Raum - , und es ist auch eine Arena, in der um Veränderung oder Erhaltung dieses Kräftefeldes gekämpft wird. In diesem Universum bringt jeder die (relative) Kraft, über die er verfügt und die seine Position im Feld und folglich seine Strategien bestimmt, in die Konkurrenz mit den anderen ein.“7

Medienerzeugnisse sind Produkte - produziert unter Marktbedingungen im Blick auf und für ein Publikum oder mehrere Publika mit bestimmten sozialen Eigenschaften (Wunschvorstellungen, Bedürfnissen, Konsumgewohnheiten, Medienkompetenzen usw.). Die Medienpublika bilden also, um mit Elias' figurationssoziologischer Terminologie zu sprechen, einen figurativen „Pol“, der in dialektischen Verhältnissen zu dem „Pol“ der Medienproduzenten steht. Diese zerfallen entsprechend der funktionalen Differenzierungslogik ihrer Handlungssphäre in verschiedene Akteursklassen, die in wechselseitigen Abhängigkeitsverhältnissen zueinander stehen und höchst „aktiv“ sind. Mit Elias kann man von „Interdependenzgeflechten“ von Akteuren und Akteursformationen sprechen, die um Markterfolge kämpfen. Deren Messbarkeit, z. B. in Gestalt der „Einschaltquote“, hat zu einer Art „Diktatur"8 geführt, die alle am Markt-Spiel Beteiligten mehr oder weniger unterwirft. Sie erzwingt korrelativ zur fortschreitenden soziokulturellen Differenzierung der Gesellschaft ein zunehmend differenziertes und zielgenaues „recipient design“ der Medienproduktion. Die an ihr beteiligten Rollen müssen sich entsprechend einstellen und differenzieren.

Unter dem alles beherrschenden Zwang stehend, sich an dem jeweiligen Publikum zu orientieren, operieren die ganz oder teilweise auf Publikumsbeobachtung spezialisierten Medienakteure mindestens „parasoziologisch“. Sie rekonstruieren in einem durch ihren Handlungszweck bestimmten Maß Aspekte der Wirklichkeitskonstruktion ihres aktuellen oder virtuellen Publikums, um handlungsrelevante Urteile und Erwartungen erwarten und dann angemessen dramaturgisch (re-)agieren zu können. ${ }^{9}$ Dieser „Perspek-

6 Schmidt 1995, S. 84.

7 Bourdieu 1998, S. 57. Ganz ähnlich (und früher) definiert Elias sein Konzept der Figuration als das „Bild vieler einzelner Menschen, die kraft ihrer elementaren Ausgerichtetheit, ihrer Angewiesenheit aufeinander und ihrer Abhängigkeit voneinander auf die verschiedenste Weise aneinander gebunden sind und demgemäß Interdependenzgeflechte [...] mit mehr oder weniger labilen Machtbalancen verschiedenster Art bilden ..." (Elias 1981, S. 12). Auch bei Elias geht es um Kräfte („Valenzen“), ungleiche Kraftverhältnisse und Konkurrenzen bzw. Kämpfe, aus denen immer wieder neue Kraftverhältnisse hervorgehen.

8 Bourdieu 1998, S. 22.

9 Das bedeutet natürlich nicht, dass die jeweilige Beobachtungsbemühung zu einem realistischen medialen Realitätsbild führt. So spricht z. B. viel dafür, dass die Werbung ein ziemlich unrealistisches Bild des Geschlechterverhältnisses zeichnet. Das in der werblichen Geschlechterdarstellung immer noch vorherrschende Sujet der Frau als „passives, abhängiges, untergeordnetes 
tivenwechsel“ erfolgt selbst in so rationalisierten und durchorganisierten Feldern wie der Werbung in hohem Maße intuitiv, d. h. auf der Basis fungierender Habitus, die als jedermanns „Innenausstattung“ und als professionelle Varianten (z. B. Geschmacks-) Urteile und Urteilskraft hervorbringen. Medienproduzenten aktivieren zudem und auf dieser Basis ein professionell-technisches „Lehrbuchwissen“.

Der Blick und die dramaturgische Anstrengung der Medienproduzenten richtet sich aber nicht nur direkt auf ihre Publika, die für sie sozusagen die letzte Instanz darstellen. Privilegierter Gegenstand der strategischen Beobachtung sind vielmehr auch die Marktkonkurrenten. So verbringen Journalisten in ihren Redaktionskonferenzen „beträchtlich viel Zeit damit, von anderen Zeitungen zu sprechen, besonders von dem, ,was sie gemacht haben und wir nicht' (,das haben wir verschlafen') und was man - selbstverständlich - hätte machen müssen, da die anderen es gemacht haben“. ${ }^{10}$ Ebenso richtet sich etwa die Beobachtung der Werbungsproduzenten immer auch auf andere Werbungsproduzenten und die Gründe für deren Erfolge und Misserfolge.

\section{Medien, Wissen, Sinn}

Eine Analyse, die versucht, Medientheatralität als einen Zusammenhang zwischen den Ebenen der Medienproduktion, der Medienprodukte („Medientexte“) und der Medienrezeption zu verstehen, braucht entsprechend formale und damit universelle Sinn- und Wissensbegriffe. Sie sind hier gefragt, weil zum einen alle Sinn- und Wissensformen, die die Kultur zu bieten hat, im Zusammenhang der genannten Medienebenen eine Rolle spielen und zum anderen die Medien selbst eine expandierende und sich diversifizierende Kultur darstellen, die nicht nur auf Kultur referiert, sondern auch in eigenen Sinnformen Kultur moduliert und generiert. Vor diesem Hintergrund erscheinen die auf Theorien verweisenden Konzepte Semantik und Rahmen besonders brauchbar, und zwar teilweise in einem komplementären Sinne.

\subsection{Semantik}

Niklas Luhmanns Semantikbegriff bezieht sich auf den gesamten gesellschaftlichen „Vorrat an bereitgehaltenen Sinnverarbeitungsregeln [...], einen höherstufig generalisierten, relativ situationsunabhängig verfügbaren Sinn“, ${ }^{11}$ der im Erleben und Handeln aktualisiert wird. Damit ist zunächst der „Alltagsgebrauch“ von Sinn gemeint. Auf dieser Ebene ist die Semantik einer Gesellschaft ausschnitthaft für jedermann verfügbar. Davon zu unterscheiden ist die Ebene der „gepflegten Semantik“. Auch sie wird im Erleben und Handeln aktualisiert, ist aber an dafür ausdifferenzierte Situationen, Rollen und Teilsysteme sowie an Schrift oder Schriftanaloga gebunden. ${ }^{12}$

Der Semantikbegriff ist so umfassend, dass er alle Wissensformen beinhaltet. Luhmanns Hauptinteresse gilt aber der gepflegten Semantik, den ausformulierten Ideen der Religion, der Kunst, der Philosophie, der Wissenschaft etc. Daneben geht es ihm um die

[...] Beiwerk des Mannes“ (Esser / Hesse 1996, S. 35) dürfte kaum noch in Übereinstimmung mit der Normalform des Geschlechterlebens in der heutigen Mittelschicht stehen (vgl. Behrens / Hagge 1990, S. 166).

10 Bourdieu 1998, S. 32.

11 Luhmann 1980, S. 19.

12 Vgl. Luhmann 1980, S. $19 f$. 
kollektiven Vorstellungen von Liebe, Sexualität, Individualität usw., die er in einer Art Sickertheorie von der gepflegten Semantik ableitet. Habituelles Alltagswissen, wie es etwa bei Elias, Bourdieu und Goffman im Vordergrund steht, kommt dagegen bei Luhmann kaum vor.

Für die Analyse der Medientheatralität, die auf allen Ebenen mit dem Semantikbegriff arbeiten kann, stellt sich also im Anschluss an Luhmann vor allem die Frage der (system-)spezifischen Verarbeitung, Tradierung und Überformung von „gepflegter Semantik" und deren Abkömmlingen. Mit Luhmann kann man etwa den Individualismus der Werbung oder die in Hochzeitsshows inszenierten Liebesvorstellungen als semantische „Ableitungsgeschichten“ rekonstruieren. Luhmann liefert zudem, weil er - wie Elias und im Gegensatz zu Bourdieu - über eine historisch orientierte Gesellschaftstheorie verfügt, einen Ansatz zum Verständnis semantischer und damit auch mediendramaturgischer Erfolge und Misserfolge. Welche Ideen sich durchsetzen, Dominanz gewinnen und kontinuieren, das hängt Luhmann zufolge von den gesellschaftlichen Strukturen ab. Der durch sie erzeugte Problemlösungsbedarf wirke als Selektionsrahmen für die Ideen. Zentrale Bedeutung spricht Luhmann dabei der Umstellung der Differenzierungsform der Gesellschaft von primär stratifikatorischer auf primär funktionale Differenzierung zu, die er für die wichtigste Variable des Modernisierungsprozesses hält. In den mit dieser Umstellung sich wandelnden sozialen Existenzbedingungen sieht Luhmann Probleme, für die die semantische Entwicklung Lösungen bereitstellen muss. Lösungen dieser Art sind in Medieninszenierungen wie z. B. den gegenwärtig ausgestrahlten Seifenopern zu sehen.

\subsection{Rabmen}

Im Unterschied und komplementär zu Luhmann fokussiert Goffman mit seinem Rahmenkonzept ${ }^{13}$ nicht die „gepflegte Semantik“, sondern genau jenen „Alltagsgebrauch“ von Sinn, den Luhmann vernachlässigt, obwohl sein Semantikbegriff ihn umfasst. Der Goffmanschen „Rahmen-Analyse“14, die sich umgekehrt auch auf die „gepflegte Semantik" erstrecken könnte, geht es um die strukturierten und strukturierenden Sinnimplikationen der Alltagspraxis als einem Erfahrungs- und Handlungsraum, in dem „Normalität" von intuitiv wissenden Akteuren vorgestellt, unterstellt und dargestellt wird. ${ }^{15}$

Goffman entwirft und beschreibt Rahmen als Sinnstrukturen, die es als Elemente von „Systemen“ ermöglichen zu erkennen und zu erhandeln, „was eigentlich los“ ist. ${ }^{16} \mathrm{Un}$ ter Goffmans systematischen Gesichtspunkten entscheidend ist die Differenzierung zwischen „primären Rahmen“ einerseits und „Moduln“ und „Täuschungen“ andererseits. ${ }^{17}$ „Primäre Rahmen“ nennt Goffman elementare Strukturen des untransformierten Sinnhintergrunds, der als Grundlage von Sinntransformationen fungiert. ${ }^{18}$ Eine

13 Neben dem Rahmenbegriff ist hier an eine Reihe weiterer Begriffe zu denken, die sich teilweise inhaltlich überschneiden oder ersetzen und teilweise ergänzen, z. B.: Diskurs, kommunikative Gattung, Sinnwelt, Skript oder Deutungsmuster. Zur wissenssoziologischen Bedeutung der Rahmentheorie vgl. Willems 1997.

14 Goffman 1977.

15 Vgl. Soeffner 1989, S. 146 ; Srubar 1994, S. $109 \mathrm{ff}$.

16 Goffman 1977, S. $12 \mathrm{f}$.

17 Vgl. Goffman 1977, S. 52ff.

18 Vgl. Grathoff 1978, S. 69; 1989, S. 291. 
Transformation eines primären Rahmens - und damit auf einer ersten „Schicht“ die Erzeugung von Sinnkomplexität - liegt z. B. vor, wenn „ein Stück Kampfverhalten in ein Stück Spiel verwandelt“ wird ${ }^{19}$ oder wenn ein Geschehen medial dokumentiert wird. Einen Rahmen, der in diesem Sinne anweist, „sekundär“ zu verstehen, nennt Goffman Modul (key). Ein Modul ist also ein Schema, durch das eine Tätigkeit, die bereits in einem primären Rahmen sinnvoll ist, in etwas transformiert wird, das dieser Tätigkeit nachgebildet ist, von den Beteiligten aber als etwas ganz anderes gesehen wird. ${ }^{20}$ Täuschungen sind demgegenüber Formen der einseitigen Sinntransformation, denen „... das bewußte Bemühen eines oder mehrerer Menschen (zugrunde liegt, H. W.), das Handeln so zu lenken, daß einer oder mehrere andere zu einer falschen Vorstellung von dem gebracht werden, was vor sich geht. (...) Es scheint also, daß ein Stück Handlung zweierlei in die Welt setzen kann, daß es als Muster dienen kann, von dem zwei Arten von Ableitungen hergestellt werden: eine Modulation oder eine Täuschung “21 . In Rahmen aller Art sieht Goffman reale Strukturtatsachen auf der Ebene der kulturellen Manifestationen (z. B. „Medientexte“) und - korrespondierend - auf der Ebene der Wahrnehmung bzw. des Wahrnehmenden. Im Prozess der Wahrnehmung wird die Wirklichkeit demnach weniger konstruiert als rekonstruiert.

Die Rahmen-Analyse erfasst die verschiedensten alltagsweltlich-habituellen Darstellungen, z. B. Grüße, Benehmen oder Takt, ebenso wie Inszenierungen in dem Sinne, dass „Handlungen oder Zusammenhänge absichtsvoll und mit einer bestimmten Wirkungsabsicht zur Erscheinung gebracht werden“. ${ }^{22}$ Inszenierungen lassen sich mit Goffman als Varianten der zwei großen Rahmenklassen (Modulationen und Täuschungen) bestimmen. Die Bandbreite reicht dabei von Modulationstypen wie der ironischen Kommunikation über „gut gemeinte“ Täuschungsmanöver wie das „Bären-Aufbinden“ bis hin zu „schädigenden Täuschungen“ wie den Formen des Betrugs. Inszenierungen finden für Goffman also keineswegs nur auf den Bühnen der Theater und der Massenmedien statt. Sie sind vielmehr Momente aller Bereiche des sozialen Lebens, insbesondere auch des Alltagslebens. Allerdings sieht Goffman im Theater und in den verschiedenen Medientypen (Film, Fotografie usw.) Rahmen eigener Art, die je besondere Inszenierungspotenziale beinhalten. Für Goffman ist dabei entscheidend - und diese Überlegung ist im Hinblick auf die im Folgenden zu behandelnde Perspektive der „Cultural Studies" von besonderer Wichtigkeit -, dass die Theateraufführungen und die massenmedialen Inszenierungen eine objektive Sinnkomplexität (Sinnschichtung) implizieren, die die Theater- und Medienakteure ebenso wie deren Publika typischerweise intuitiv verstehen. Dass das normale (Theater-, Medien-) Publikum höchst sinnkomplexen, rahmenverschachtelten Aufführungen anhand entsprechender „RahmungsHinweise“ folgen kann, hält Goffman für eine „große Lektion, und sie sagt uns etwas über eine wichtige menschliche Fähigkeit, die in Bezug auf wirkliche wie auch fiktive Ereignisse zum Tragen kommt ${ }^{\text {“23 }}$. Ein Beispiel für diese Fähigkeit, d. h. für die Möglichkeit und Wahrscheinlichkeit einer „Parallelführung“ von Enkodierung und Dekodierung, Text und Wahrnehmung (Verstehen), ist das Folgende, das Goffman von dem Filmkritiker Bela Balasz übernommen hat:

19 Goffman 1977, S. 52.

20 Goffman 1977, S. $55 f$.

21 Goffman 1977, S. 98.

22 Ontrup/ Schicha 1999, S. 7.

23 Goffman 1977, S. 208. 
„Asta Nielsen [eine deutsche Schauspielerin] spielte einmal eine Frau, die man dazu gedungen hatte, einen reichen jungen Mann zu verführen. Der Mann, der sie dazu zwingt, beobachtet sie, hinter einem Vorhang versteckt, und wartet auf das Ergebnis. Im Bewußtsein dieser Kontrolle täuscht Asta Nielsen Verliebtheit vor. Sie tut es überzeugend, ihr Antlitz spiegelt die ganze mimische Skala der Liebe. [...] Wir aber sehen, daß es nur gespielt ist, unecht - nur eine Maske. Im Verlauf der Szene nun verliebt sich Asta Nielsen wirklich in den jungen Mann. Ihr Mienenspiel ändert sich kaum, hatte es doch auch bisher Liebe gezeigt - und das vollkommen! Was sonst könnte es also jetzt, da sie wahrhaft liebt, zeigen? Es ist nur um jenen kaum faßbaren und dennoch gleich erkennbaren Schimmer anders, der bewirkt, daß jetzt der Ausdruck echten, tiefen Gefühls wird, was vorher Verstellung war. Der Mann hinter dem Vorhang darf in ihrer Miene nicht lesen, daß es kein Spiel mehr ist, was sie treibt. Sie tut also wieder, als lüge sie. Auf ihrem Antlitz erscheint eine neue, eine nunmehr dreistimmige Variation. Denn ihr Mienenspiel täuschte erst Liebe vor und zeigte sie dann aufrichtig. Letzteres aber darf es nicht. Also zeigt ihr Gesicht wieder falsche, vorgespiegelte Verliebtheit. Sie lügt uns vor, daß sie lügt. Und das alles sehen wir deutlich auf Asta Nielsens Gesicht. Sie hat zwei mimische Masken übereinandergelegt! Ein unsichtbares Antlitz wird nun auf ihrem Gesicht sichtbar (ähnlich wie gesagte Worte oft durch Assoziation das Unsagbare hervorrufen). Das sichtbare Mienenspiel assoziiert das unsichtbare, das nur derjenige versteht, dem es gilt.'

Doch natürlich stammt die ganze Szene aus einem Film, und die Menschen, die ihn spielen, haben weder die Absicht noch die Erwartung, das Publikum in ein Mißverständnis hineinzumanövrieren; Fräulein Nielsen verhält sich so, daß dem Publikum völlig klar wird, daß sie das Vorlügen einer Lüge spielt, und irgendwie bekommen die Zuschauer genügend Hinweise, um die Schichten ohne weiteres ablösen zu können“. ${ }^{24}$

Inszenierungen lassen sich also jeweils als Rahmentyp bzw. Rahmenkomplex beschreiben, dem ein zumindest potenziell angemessenes Verstehen, und d. h. ein auf den (Eigen-) Sinn der Inszenierung bezogenes „Lesevermögen“ („Rahmen-Literalität“), korrespondiert.

Wahrnehmung, Umgang mit Wahrgenommenem und performativ-aktive Umsetzung von Sinn bedeuten für Goffman allerdings keineswegs in strukturalistischer Manier bloße Kopiervorgänge. Vielmehr geht Goffman von einer prinzipiellen Differenz von Sinnstruktur und Praxis aus. In diesem Sinne belegt er die Ebene der Sinnstruktur mit dem Begriff des Rahmens und die Ebene der kognitiven oder performativen Aktualisierung der Sinnstruktur mit dem der Rahmung. Während sich für Goffman Rahmen durch relative Autonomie und Immunität gegenüber der Praxis auszeichnen, ist die Rahmung, die Umsetzung von Sinn und der Sinn für Sinn, für ihn mehr oder weniger kontingent, offen und anpassungsbedürftig. Rahmen stellen für Goffman also keine starren Regelsysteme dar, die das Handeln und Wahrnehmen der Akteure determinieren, sondern eher elastische und „improvisationsfähige“ Strukturen, die mehr oder weniger viel Raum für Variationen, Anpassungen und Konkretisierungen lassen. Die entschieden antideterministische, Dialektiken von Grenzen und Spielräumen betonende Position Goffmans bringt Phil Manning auf folgende Formulierung: „There will never be a book for the rules of social life that is analogous to a book for the rules of chess because it is impossible to specify all contexts and all the possible moves open to interaction. ${ }^{25}$ 
Goffman argumentiert damit im Prinzip wie Bourdieu. Auch dieser weist die Vorstellung einer mechanischen Determiniertheit der Praxis durch die ihr zugrunde liegenden Schemata zurück:

„Um zu ermessen, auf welche Schwierigkeiten eine mechanistische Theorie der Praxis stieße, bei der diese als eine rein mechanische Reaktion definiert würde [...] braucht man sich nur das grandiose und verzweifelte Vorhaben jenes Ethnologen vorzustellen, der mit schönstem positivistischen Wagemut nach zwanzigminütiger Beobachtung der Küchenarbeit seiner Frau 480 elementare Verhaltenseinheiten aufzeichnet und dann die ,Episoden', die wissenschaftlich verarbeitet werden müssen, auf 20000 pro Tag und pro Akteur, also für eine Gruppe von mehreren Hundert Klassen von Akteuren auf mehrere Millionen jährlich schätzt. “26

Wenn nun aber die konkrete Komplexität der Praxis niemals strukturell determiniert sein kann und jede praktisch zu aktualisierende Sinnstruktur relativ offen sein muss, dann muss auch eine soziale und sozial erzeugte „Instanz“ im Individuum existieren, die leistet, was die Struktur selbst nicht leisten kann. Diese „Instanz“, die Urteilsvermögen, Flexibilität und Kreativität hervorbringt, konzipiert der Habitusbegriff. ${ }^{27}$ Als Kompetenzbegriff ergänzt er den Rahmenbegriff, der mit seiner Betonung der Offenheit von Sinnstrukturen in der Nähe einer medienanalytischen Grundposition der „Cultural Studies" steht.

Die medientheoretische und medienanalytische Relevanz des Rahmenkonzepts erweist sich zudem in folgenden Punkten:

a) Medienerzeugnissen (z. B. der Tagespresse) sind mit den Mitteln der Rahmen-Analyse die alltagsweltanschaulichen (kosmologischen) Sinngehalte zu entnehmen, die ihre Produzenten - im Blick auf ihre Publika - in sie „hineingesteckt “ ${ }^{\text {28 }}$ haben. Die verschiedensten Medienerzeugnisse (von der Werbung bis zu den Nachrichten) lassen sich als „Rahmen-Fantasien (betrachten, H. W.), denen es kraft der hundert Freiheiten, die sich ihre Erzähler nehmen, gelingt, unsere Vorstellungen über den Weltlauf zu verherrlichen". 29

b) Medienerzeugnisse können als Genre-Rahmen differenziert werden, die mehr oder weniger verbindlich besagen, „welchen Status das Ganze (kommunikative Geschehen, H. W.) eigentlich in der äußeren Welt hat" ${ }^{30}$ Genres wie Werbung oder Nachrichten sind in diesem Sinne mehr oder weniger verfestigte und kollektiv gewusste Definitionen dessen, was (im Medium) „eigentlich vorgeht“. Es handelt sich um Sinn- und Wissenstypen, die von einer Meta-Ebene aus Verstehen anweisen und Erwartungen konfigurieren. Jeder Genre-Rahmen offeriert eine „ganze Grammatik von Erwartungen“, 31 die Erfahrungen und Verhaltensweisen organisieren. Zur „Gesellschaftsfähigkeit“ jedermanns gehört eine entsprechende Kenntnis und Urteilsfähigkeit. Auf der Ebene der Genre-Rahmen gibt es also nur sehr begrenzte Offenheiten und Spielräume für einen „aktiven“ Umgang mit Medienerzeugnissen.

26 Bourdieu 1987, S. 115.

27 Den Habitusbegriff habe ich an anderer Stelle ausführlich diskutiert (vgl. Willems 1997; 1997a).

28 Goffman 1977, S. 24.

29 Ebd.

30 Goffman 1977, S. 96.

31 Goffman 1977, S. 339.

32 Im Anschluss an Jörg Bergmann und Thomas Luckmann kann man in diesem Zusammenhang auch - statt von Rahmen - von „kommunikativen Gattungen“ sprechen. Beide Begriffe eröff- 
c) Medienerzeugnisse lassen sich als Genre-Rahmen im Hinblick auf ihre je besonderen Potenziale und Kontingenzspielräume der Rahmung und Rahmenschichtung, z. B. Modulationsmodulation, bestimmen. Jeder Genre-Rahmen etabliert ein spezifisch strukturiertes Feld der Sinnvariation und Sinnstrukturierung. Der Genre-Rahmen der Pornografie z. B. eröffnet einen variablen Raum von Obszönität innerhalb von Grenzen, die die Identität des Genre-Rahmens setzt. ${ }^{33}$ Medienerzeugnisse lassen sich also im Prinzip ähnlich wie Gespräche - als gerahmte „Ströme verschieden gerahmter Abschnitte ${ }^{34}$ beschreiben.

d) Die Rahmen-Analyse kann sich aber auch auf die Variabilität der Genregrenzen (des „Rahmenrandes“) beziehen. Sie übersieht keineswegs - auch wenn sie eine historische Gesellschaftstheorie von der Art der Elias'schen entbehrt -, dass die Grenzen der Genres und Subgenres Entwicklungsgeschichten haben und, wenn auch typischerweise langfristig, (Grenz-)Wandlungen unterworfen sind. Ein Beispiel findet sich in der Rahmen-Analyse der "Sonderausführung“, wo Goffman ${ }^{35}$ folgende Pressemeldung (New York Times, 5. 8. 1957) zur Illustration einer historischen Rahmengrenzverschiebung zitiert:

„Toronto, 4. Aug. (Canadian Press) - Die Kanadische Rundfunk- und Fernsehgesellschaft hat das Verbot der Werbung für Artikel aufgehoben, die bisher als zu intim für das Fernsehen gegolten hatten. Werbung für Korsetts, Desodorierungsmittel, Büstenhalter, Fitnessklubs, Haarentferner und Toilettenpapier kann jetzt Eingang ins Fernsehen finden. ,Themen, die noch vor ein paar Jahren in gemischter Gesellschaft als unschicklich galten, werden jetzt als akzeptabel empfunden', sagte Charles Spraggett, der Leiter der Presseabteilung der CBC. Damenslips bleiben ausgeschlossen. “ ${ }^{6}$

e) Die Rahmen-Analyse erfasst auch Formen der medialen Sinnverarbeitung, d. h. (,intertextuelle“, „interkontextuelle“) Relationen von Medientexten zu anderen Medientexten und $\mathrm{zu}$ „Texten“ der lebensweltlichen Alltagspraxis. Besonders interessant ist die von Goffman exemplarisch vorgeführte Möglichkeit, genrespezifische Verarbeitungen von (Ritual-)Rahmen zu analysieren, die dem alltäglichen Interaktionsleben zugrunde liegen. So erkennt er in der Werbung „Hyper-Ritualisierungen“, die in rahmenspezifischer Weise alltägliche Ritualisierungen (der Berührung, der Kleidung, der instrumentellen Tätigkeit usw.) kopieren. ${ }^{37}$

nen „die Möglichkeit, das kommunikative Geschehen im Fernsehen und in anderen Massenmedien als massenmedialen kommunikativen Haushalt einer Gesellschaft zu erfassen, als ein Teil jenes umfassenden kommunikativen Haushalts, dessen wir uns tagtäglich bedienen" (Ayaß 1999, S. 3).

33 Vgl. Willems 1997, S. $412 \mathrm{ff}$.

34 Goffman 1977, S. 584f.

35 Goffman 1977, S. 71ff.

36 Goffman 1977, S. 81. Eine neuere Entwicklung betrifft die Genre-Rahmen des Fernsehens, deren Wandlungen sich im Bereich der narrativen Texte besonders in der Übernahme von „Darstellungs- und Inszenierungstechniken aus anderen Genres zeigen. Ein extremes Beispiel hierfür stellt die amerikanische Serie ,Twin Peaks' dar, bei der das Überschreiten von Genregrenzen und die Pastichebildung aus Stilelementen verschiedenster Genres in postmoderner Manier geradezu zum Programm gemacht wurde“ (Jurga 1998, S. 111).

37 Vgl. Goffman 1981. 


\section{Mediale Foren}

Die „gesellschaftliche Konstruktion der Wirklichkeit“ ist heute im Wesentlichen massenmediale Konstruktion. Oder mit Niklas Luhmann formuliert: „Was wir über die Gesellschaft, ja über die Welt, in der wir leben, wissen, wissen wir durch die Massenmedien. "38 Sie sind die gigantischen Raum-, Zeit- und Sozial-Grenzen überspringenden „Bühnen“, auf denen kulturelle Sinnbestände und Informationen (re-)präsentiert, verarbeitet und verbreitet werden. Wie zahlreiche andere Medientheoretiker betrachten Newcomb und Hirsch das Fernsehen als das gegenwärtig zentrale „kulturelle Forum“, das ein Massenpublikum mit der „Vielfalt von Ideen und Ideologien unserer Kultur konfrontiert “39. Ähnlich wie Newcomb und Hirsch sehen Goffman ${ }^{40}$ und Fiske/ Hart${ }{ }^{41}{ }^{41}$ in Massenmedien reflexive und zugleich theatrale Instanzen, die Kultur selektiv aufgreifen und „aufführen“. Fiske und Hartley konstruieren die kulturelle Funktion des Fernsehens analog zu der Rolle des Barden in mittelalterlichen Gesellschaften. Entscheidend ist in diesem Zusammenhang die Annahme, dass die Erzählungen des Barden wie die Kommunikationen des Massenmediums von der Adressierung an ein bestimmtes Publikum bestimmt sind. Sie müssen in der „Sprache“ des Publikums formuliert sein, d. h. auch im Rahmen von dessen Relevanzstrukturen, Bedürfnissen, Wunschvorstellungen usw. stehen. „The real authority for both bardic and television messages is the audiences in whose language they are encoded. “42 Man kann in diesem Zusammenhang analog zum alltäglichen „recipient design“ der Konversation in Face-to-Face-Kontexten von einem „medialen recipient design“ sprechen ${ }^{43}$. Der Zwang zu diesem „Design“ hat sich historisch zunehmend verallgemeinert, verschärft und pluralisiert, was mit Prozessen der Differenzierung und Expansion von Märkten, Konkurrenzen und Publika zusammenhängt. Die Medienproduktion muss sich immer mehr, immer bewusster und immer gezielter auf immer diversere Publika einstellen, deren Erwartungen erwarten und Wahrnehmungen wahrnehmen. Das wie eine Währung generalisierte und formalisierte Erfolgskriterium ist dabei jener sich zunehmend verfeinernde Typ von Messung, der mit dem Begriff Einschaltquote belegt ist. In ihr spiegelt sich analog zum Applaus des Theaterpublikums Zuschauerinteresse und damit vor allem Werberelevanz. Im Hinblick auf das Feld des Journalismus spricht Bourdieu von der „Einschaltquotenmentalität“. Ihr gelte als „Maßstab der Verkaufserfolg [...] der Markt mehr und mehr als Legitimationsinstanz “44. Dementsprechend tendiert das „kulturelle Forum“ der Massenmedien „zu einer Zensur, die ebenso wirksam ist wie die einer zentralen Bürokratie, eines förmlichen politischen Eingriffs, ja wirksamer noch, weil unauffälliger “. ${ }^{45}$ Unauffälliger ist diese Zensur, weil sie nicht einer Logik des Verbots und der Untersagung, sondern des Gefallens folgt. Die systematische Exklusion von Inhalten und (z. B. Reflexions-) Formen resultiert aus dem notwendigen oder zumindest nahe liegenden Bestreben und

38 Vgl. Luhmann 1996, S. 9.

39 Newcomb/ Hirsch 1986, S. 183.

40 Goffman 1977; 1981.

41 Fiske/ Hartley 1978.

42 Fiske/ Hartley 1978, S. 86.

43 Ayaß 1999, S. 5.

44 Bourdieu 1998, S. 36.

45 Bourdieu 1998, S. 34. 
der Kunst, dem Publikum zu gefallen. Diese Kunst führt in gewisser Weise zur Verdoppelung der „Grammatik“ der Publikumskultur, die in endlosen Oberflächenvariationen gleichsam in die Medienerzeugnisse „hineingelegt“ (enkodiert) wird, damit sie von dem entsprechend adressierten Publikum „herausgeholt“ (dekodiert) werde. Die Medienerzeugnisse sind $\mathrm{m}$. a. W. die von den Medienproduzenten vorweggenommene „Lieblingslektüre“ ihres Publikums.

In der Medienkommunikation verlaufen „Enkodierungs-“ und „Dekodierungsprozesse“ demnach - gerade unter Konkurrenzbedingungen der Medienproduzenten keineswegs unabhängig voneinander. Sie sind vielmehr systematisch, systemisch und strategisch miteinander verquickt. Die Habitus der Produzenten, speziell diejenigen, die sie mit ihren Publika teilen, spielen dabei eine maßgebende Rolle, weil und insofern sie das der Medienproduktion und den Medienprodukten zugrunde liegende „Lesen“ des Publikumslesens anleiten. Letzteres hängt primär von den habituellen „Wahrnehmungs-, Denk- und Handlungskategorien der Rezipienten ab, so daß in einer hochdifferenzierten Gesellschaft eine enge Beziehung zwischen der Natur und Qualität der ausgesandten Information und der Struktur des Publikums besteht. Ihre Lesbarkeit und Durchschlagskraft sind umso größer, je direkter sie auf implizite oder explizite Erwartungen antworten, die die Rezipienten prinzipiell ihrer Erziehung durch das Elternhaus und ihren sozialen Bindungen [...] verdanken“. ${ }^{4}$

Die Medienproduzenten müssen ihre Inszenierungen aber nicht nur, unter einem zunehmenden Zwang zur perspektivischen und dramaturgischen Differenzierung stehend, den Dispositionen ihrer Publika unterordnen, sondern auch den Gesetzen des jeweiligen Genres, mit dem sie es zu tun haben. Es bildet immer auch als Teil des habituellen Publikumswissens den Rahmen, in dem das Publikum dramaturgisch „angesprochen“ werden kann und der die Form der dramaturgischen Publikumsansprache strukturiert.

\section{Medienrezeptionen: Kontexte und Kompetenzen}

Wenn man die Massenmedien in dem beschriebenen Sinn als kulturelles Forum bzw. als Ensemble kultureller Foren betrachtet, dann ist davon auszugehen, dass die entsprechenden Medienerzeugnisse viel mit dem habituellen Sinn des jeweils anvisierten Publikums zu tun haben. Dieser Sinn wandert durch die intuitiven Erwartungserwartungen der Medienproduzenten sowie durch deren strategisch gezielte Publikumserforschung gleichsam in die Medienprodukte ein. Damit ist aber nicht gesagt, dass diese immer „dekodiert“ werden wie sie „enkodiert“ worden sind. Im Hinblick auf Fernsehtexte stellt Werner Holly grundsätzlich fest:

„Die Rezipienten und Rezipientinnen sind alles andere als Sklaven eines Fernsehtextes mit einer einzigen Lesart. Wie genau diese eigenständigen Rezeptionsweisen und Deutungen verlaufen, mit welchen Faktoren des situationellen und subkulturellen Kontextes sie zusammenhängen, davon wissen wir allerdings kaum etwas. “47

Im Anschluss an die Cultural Studies, die die spezifischen Merkmale der Relationen von Medientexten und Medienrezeptionen aufdecken wollen, ist jedenfalls von einem vielfältig aktiven und produktiven Umgang der Medienrezipienten mit den von ihnen wahrgenommenen Medienerzeugnissen auszugehen, und zwar abhängig von soziokulturellen Positionierungen und Sozialisationsschicksalen der wahrnehmenden Subjekte. 
In den Vordergrund treten im Rahmen dieser Forschungstradition Spielräume der Bedeutungskonstitution im Prozess der Aneignung medialer Texte. Zentral ist in diesem Zusammenhang die Vorstellung der Offenheit. Gemeint sind damit Phänomene auf allen Ebenen des medialen Prozesses, soweit sie (Be-)Deutungsspielräume induzieren.

„Bedeutsam für das Verständnis des Offenheitsphänomens sind daher sowohl die Textseite, also gleichsam die materielle Seite der Kommunikation, als auch die Rezipientenebene bzw. die Beziehungen, die die Rezipienten mit dem Text aufbauen (...). Im Kern geht es aber nicht nur um die Feststellung kontingenter Strukturen auf der Textund Zuschauerseite, sondern um das Ineinandergreifen dieser beiden Instanzen, also um einen kommunikativen Prozeß, der zwischen ihnen stattfindet“" ${ }^{48}$

Die Cultural Studies und die ihnen verwandte Forschungstradition des Symbolischen Interaktionismus konvergieren mit ihrer Vorstellung von Offenheit sowie mit den damit verbundenen Verständnissen der Subjektivität, der Situativität und der Kontextualität der Bedeutungskonstitution einerseits deutlich mit der Rahmen-Analyse. Die Annahme einer relativen Autonomie der Rezeption und des Rezipienten, für den Medienerzeugnisse, systemtheoretisch gesprochen, Umwelten sind, die er „autopoietisch“ konstruiert und „dekonstruiert", teilt Goffman ebenso wie die der potenziellen „Polysemie“ medienkultureller Manifestationen. Andererseits wendet sich Goffman gegen einen so weit gehenden Konstruktivismus wie er die Arbeiten der Cultural Studies und des Symbolischen Interaktionismus typischerweise kennzeichnet. Mit dem Rahmenbegriff geht er stattdessen von Wirklichkeiten stiftenden Sinnstrukturtypen als spezifischen Verhältnissen von Geschlossenheit (Begrenztheit, Restriktion) einerseits und Offenheit andererseits aus. Dies impliziert die Annahme einer Autonomie der Praxis, in der die Akteure gezwungen und befähigt sind, die Offenheiten der Sinnstrukturen interpretativ zu schließen, unter Umständen aber auch die Geschlossenheit von Sinnstrukturen interpretativ zu öffnen. Entscheidend ist, dass Goffman auf einem Mittelweg zwischen Realismus und Konstruktivismus ${ }^{49}$ einen Ansatz zur Analyse von Sinnstrukturen, Kontingenzspielräumen und Sinnstrukturierungen liefert, der Subjektivitäts- und soziale Situationsaspekte mit einbezieht, ohne subjektivistisch und situationistisch zu sein.

Die praktische Interpretation (auch) von Medientexten ist für Goffman damit zunächst eine Frage des anwendbaren oder angewandten Rahmentyps oder Rahmensystems, aus dem sich je besondere Wirklichkeits- oder Weltbilder ergeben. In zweiter Linie geht es ihm um die Offenheit und Anpassbarkeit der Rahmung als Rahmenanwendung und als selektive Handhabung von Rahmen durch Rahmungssubjekte, deren „Deutungsaktivitäten“ sich prinzipiell an jeder „Materialität“ festmachen können. Innerhalb der Spielräume der Rahmensysteme und einzelnen Rahmen entfaltet sich für Goffman so etwas wie eine individuelle Rahmungshoheit, die der „informationellen Selbstbestimmung“ des Wahrgenommenen (z. B. Darstellers) Grenzen setzt. In der Kontinuität seiner am Theatermodell orientierten Unterscheidung zwischen dem Eindruck, den jemand zu „machen“ versucht, und dem Ausdruck, den er „ausstrahlt“, zeigt Goffman, dass die eigene und die fremde Fremdbestimmung als strukturierter Rahmungsspielraum systematisch kontingent und (rahmen-)relational ist. In der „RahmenAnalyse des Gesprächs“ heißt es:

48 Jurga 1997, S. 129.

49 Vgl. Collins 1980. 
„Denn bei der - wenn auch noch so genauen - Wahrnehmung dessen, was der Sprecher hervorrufen möchte, kann sich der Zuhörer dem Berührtsein entziehen und stattdessen das Gehörte hinaufmodulieren, es in ein Ganzes auflösen, er kann die Aussage allein von der Seite nehmen, daß sie lediglich eine versteckte Selbsterhöhung ist, oder ein müder Versuch der Schmeichelei, oder die x-te Wiederholung einer Geschichte, die man von dem Sprecher schon oft gehört hat, oder ein interessanter Versuch, sich einen vornehmen Akzent zuzulegen. [...] So kann den rasch wechselnden Rahmen in jemandes Rede ein weiteres System von Rahmenwechseln überlagert sein, die der Zuhörer hereinbringt - manchmal auch nur für sich selbst. Was der Polymorphie die Perversion hinzufügt." 50

Die Überlegung, die hier deutlich wird, ist alles andere als eine mikrosoziologische Petitesse, deren Geltung sich auf die Ebene der Alltagssituationen beschränkt. Was Goffman für den interaktionellen Zuhörer feststellt, kann ohne weiteres generalisiert werden. Es gilt für alle Rezipiententypen: Sie sind im Rahmen ihrer je besonderen, immer auch gruppenspezifischen Rahmungsmöglichkeiten in der Lage und aus kontextspezifisch praktischen Gründen geneigt, Darstellungen „hinauf-“ oder „herunterzumodulieren“. ${ }^{51}$ Auf der Basis dieser Feststellung lässt sich auch die medientheoretische Schlüsselfrage prinzipiell beantworten, ob die Rezipienten jenseits der dafür vorgesehenen „Leerstellen“ des von ihnen wahrgenommenen („primären“) Textes „nicht auch ganz autonom Offenheiten herstellen, indem sie - an Stellen, an denen der Text dies nicht gezielt anbietet, - als eine Art „semilogischer Guerilla“ (Eco) den semiotischen Überschuß nutzend den Text aufbrechen und abweichende Lesarten schaffen “52. In der Tat, so lässt sich im Anschluss an Goffman schlussfolgern, stellen Rezipienten ganz autonom Offenheiten (Kontingenzen) her und schaffen „abweichende“ Lesarten. Allerdings folgen sie dabei der Nomie des Rahmens, den sie anwenden, ohne ihn erfunden zu haben. Es kommt also darauf an, sich „ein Bild von dem oder den Rahmen einer Gruppe, ihrem System von Vorstellungen, ihrer ,Kosmologie' zu machen (...), obwohl das ein Gebiet ist, das auch genaue Analytiker gewöhnlich gern an andere weitergereicht haben “. 53

\section{Literatur}

Ayaß, Ruth (1999), Fernsehgattungen in der Aneignung, unveröffentlichtes Manuskript, Universität Gießen.

Behrens, G.; K. Hagge (1990): Werbung und Gesellschaft. Das Bild des Mannes in der Werbung, in: Werbeforschung und Praxis, Folge 5, S. $164-168$.

Bourdieu, Pierre (1982): Die feinen Unterschiede: Kritik der gesellschaftlichen Urteilskraft, Frankfurt a. M.

Bourdieu, Pierre (1987): Sozialer Sinn. Kritik der theoretischen Vernunft, Frankfurt a. M.

50 Goffman 1977, S. 588.

51 Ein schönes Beispiel für eine Form der Heruntermodulation und „Rahmungsperversion“ in der Wahrnehmung jenseits der von Goffman fokussierten Interaktionsebene findet sich in der Sadomasochismus-Studie von Wetzstein u. a. Sie berichten von einem pornographieinteressierten Sadomasochisten, der regelmäßig den neuesten Report der „Bundesprüfstelle für jugendgefährdende Schriften“ liest, um sich so über attraktive Angebote auf dem Laufenden zu halten (vgl. Wetzstein u. a. 1993, 120).

52 Holly 1995, S. 121.

53 Goffman 1977, S. 37. 
Bourdieu, Pierre (1991): Soziologie der symbolischen Formen, Frankfurt/Main.

Bourdieu, Pierre (1998): Über das Fernsehen, Frankfurt/Main.

Collins, Randall (1980): „Erving Goffman’s Sociology: Social Origins of an American Structuralism“, in: J. Ditton (Hg.), The View from Goffman, New York, S. 170 - 209.

Elias, Norbert (1980): Über den Prozeß der Zivilisation. 2 Bde., Frankfurt a. M.

Elias, Norbert (1981): Was ist Soziologie? München.

Esser, Willy; Hesse, Andreas (1996): Inszenierungen und Erzengungen geschlechtsspezifischer Verbaltensstile in Werbung und Gesellschaft (unveröffentlichte Diplomarbeit, Universität Trier).

Fischer-Lichte, Erika (1998): Inszenierung und Theatralität, in: Willems, Herbert/ Jurga, Martin (Hg.): Inszenierungsgesellschaft. Ein einführendes Handbuch, Opladen, S. 81 - 93.

Fiske, John; Hartley, John (1978): Reading Television, London.

Goffman, Erving (1977): Rabmen-Analyse. Ein Versuch über die Organisation von Alltagserfahrungen, Frankfurt a. M.

Goffman, Erving (1981): Geschlecht und Werbung, Frankfurt a. M.

Goffman, Erving (1994): Interaktion und Geschlecht, Frankfurt a. M., New York.

Grathoff, Richard (1978): Alltag und Lebenswelt als Gegenstand der phänomenologischen Sozialtheorie, in: Hammerich, K. / Klein, M. (Hg.): Materialien zur Soziologie des Alltags (Sonderheft 20 der Kölner Zeitschrift für Soziologie und Sozialpsychologie), Opladen, S. 67 - 85.

Grathoff, Richard (1989): Milieu und Lebenswelt, Frankfurt/ Main.

Holly, Werner (1995): „,Wie meine Tante Hulda, echt'. Textoffenheit in der Lindenstraße als Produktions- und Rezeptionsphänomen“, in: Jurga, Martin (Hg.), Lindenstraße. Produktion und Rezeption einer Erfolgsserie, Opladen, S. 117 - 139.

Jurga, Martin (1997): Texte als [mehrdeutige] Manifestation von Kultur: Konzepte von Polysemie und Offenheit in den „Cultural Studies“, in: Hepp, Andreas; Rainer Winter (Hrsg.): KulturMedien - Macht. „Cultural Studies“ und Medienanalyse, Opladen, S. 127-142.

Jurga, Martin (1998): Fernseh-Textualität, unveröffentlichtes Dissertationsmanuskript, Universität Trier.

Luhmann, Niklas (1980): Gesellschaftsstruktur und Semantik: Studien zur Wissenssoziologie der modernen Gesellschaft, Bd. 1, Frankfurt a. M.

Luhmann, Niklas (1996): Die Realität der Massenmedien, 2. erweiterte Auflage, Opladen.

Manning, Peter K. (1992): Organizational Communication, New York.

Newcomb, Horace M. / Hirsch, Paul (1986): „Fernsehen als kulturelles Forum. Neue Perspektiven für die Medienforschung“, in: Rundfunk und Fernsehen 34. Jg., H. 2, S. 177 - 190.

Ontrup, Rüdiger; Schicha, Christian (1999): Die Transformation des Theatralischen - eine Einführung, in: Ontrup, R.; Schicha, C. (Hg.): Medieninszenierungen im Wandel. Interdisziplinäre Zugänge, Münster, S. 7 - 19.

Sandbothe, Mike (1998): Theatrale Aspekte des Internet: Prolegomena zu einer zeichentheoretischen Analyse theatraler Textualität, in: Willems, H. / Jurga, M. (Hg.): Inszenierungsgesellschaft. Ein einfübrendes Handbuch, Opladen, S. $583-597$.

Schmidt, Siegfried J.; Spiess, Brigitte (Hg.) (1995): Werbung, Medien und Kultur, Opladen.

Schmidt, Siegfried J.; Spiess, Brigitte (1996): Die Kommerzialisierung der Kommunikation. Fernsebwerbung und sozialer Wandel 1956-1989, Frankfurt a. M.

Soeffner, Hans-Georg (1989): Auslegung des Alltags - Der Alltag der Auslegung, Frankfurt a. M.

Srubar, Ilja (1994): Lob der Angst vorm Fliegen. Zur Autogenese sozialer Ordnung, in: Sprondel, W. M. (Hg.): Die Objektivität der Ordnungen und ibre kommunikative Konstruktion, Frankfurt a. M., S. $95-121$.

Wetzstein, Thomas A. u. a. (1993): Sadomasochismus. Szenen und Rituale, Reinbek.

Willems, Herbert (1997): Rabmen und Habitus. Zum theoretischen und methodischen Ansatz Erving Goffmans: Anschlïsse und Anwendungen, Frankfurt a. M.

Willems, Herbert (1997a): Rahmen, Habitus und Diskurse. Zum Vergleich soziologischer Konzeptionen von Praxis und Sinn, in: Berliner Journal für Soziologie 7, S. 87 - 107. 